\title{
Telehealth is here to stay
}

\author{
Telehealth has emerged as an unexpected silver lining of the COVID-19 pandemic, improving access to care and \\ facilitating a transition toward digital medicine. Cementing these gains now could help make healthcare more \\ equitable once the pandemic has ended.
}

T he potential of telehealth-the use of telecommunications to enable remote visits with a doctor-for improving access to healthcare has been put into the spotlight during the COVID-19 pandemic, when in-office visits became almost impossible.

In the United States, the federal government stepped in at the beginning of the pandemic, in March 2020, to lower barriers to the use of telehealth, including removing geographical restrictions, expanding the types of services that could be provided virtually, and providing reimbursement for virtual visits equivalent to that for in-office visits. The shift to telehealth in the United States during the pandemic has been dramatic. Whereas in January 2020 , less than $1 \%$ of primary care visits in Medicare occurred virtually, by April, nearly half did. In the fall of 2020, nearly two thirds of Medicare beneficiaries reported that their provider offered telehealth appointments, up from $18 \%$ from before the pandemic. According to a market report, there were ten times more telehealth visits in March 2021 than in March 2020, and the number of covered telehealth visits at the health insurer UnitedHealth Group rose from 1.2 million visits in 2019 to 34 million in 2020 . Telehealth has also taken off in the UK and in other European countries, including Germany, France and Norway.

Relative to its ability to improve access in high-income countries such as the United States and those in Europe, the ability of telehealth to increase access to healthcare is potentially even greater in low- and middle-income countries, where there can be severe geographical barriers to access to in-person healthcare.

Commercial interest in telehealth has boomed, with both start-ups and established companies, such as Amazon, providing virtual care services. An area of particular activity has been behavioral and mental health, where multiple companies are vying to provide remote counseling. In one prominent example, the city of Reno, Nevada, instituted a $\$ 1.3$ million experimental program in which the city paid for virtual therapy of residents through the smartphone app Talkspace.

In addition to improving access to care, telehealth has spurred the use of wearable and digital technologies that enable virtual care. Remote monitoring tools, including mobility trackers, blood pressure monitors and glucometers, allow doctors to monitor the health of their patients and their response to treatment. Patients engage with smartphone health apps that can keep track of other aspects of daily living that affect their health, such as nutrition data. The detailed and long-term monitoring that these tools provide can identify early signs of disease and has the potential to improve preventative care, as well as providing more personalized care.

In realizing the ambitions of telehealth, a host of challenges will need to be faced. At this early stage in the development of this field, it remains unclear which conditions and treatments telehealth and mobile apps are effective for. Clinical studies are needed to figure out what works and what does not. In particular, given the growing use of telehealth for mental and behavioral healthcare, the efficacy of telehealth in these settings needs to be rigorously tested. For example, a recent clinical trial found that an intervention using interactive text messages was effective in prompting young adult users of e-cigarettes to stop smoking.

Equity concerns are also of paramount importance. Even in high-income countries, access to a smartphone or computer is not universal, and a recent study found that communities with higher poverty rates reported much lower telehealth utilization. Expansion of high-speed broadband internet access to underserved communities and outreach efforts are needed to ensure that telehealth does not exacerbate health inequities. Audio-only telehealth has been proposed as a back-up option for people without access to or ability to use audiovisual technology.

Patient privacy and data security will also need to be ensured for telehealth to be viable. A recent study found that health apps have inconsistent privacy practices and that a substantial fraction of these apps do not have an explicit privacy policy. The possibility of such fraud was highlighted in June 2020, when there was a data breach at the UK-based telehealth company Babylon Health.

In the United States, the measures that were enacted at the beginning of the pandemic to facilitate access to telehealth will expire once the COVID-19 public-health emergency has ended, and federal legislation is needed to ensure that the potential of telehealth is realized. A number of bills are under consideration that have bipartisan support, including the CONNECT for Health Act and the Telehealth Modernization Act, which would permanently remove geographic restrictions on telehealth and expand the types of services that can be provided. These acts do not yet allow doctors to practice telehealth in states in which they are not licensed, a bureaucratic barrier that should also be addressed in this legislation. Another looming debate is whether the government will continue to reimburse telehealth visits at the same rate as that for in-person visits.

By enabling remote access to care for patients regardless of where they are located, telehealth has the potential to push the healthcare system to become more equitable. As the use of telehealth expands, research into telehealth outcomes must keep up to ensure that it is used in ways that help the patient, and measures must be taken to ensure that telehealth contributes to rather than detracts from health equity.

Published online: 15 July 2021 https://doi.org/10.1038/s41591-021-01447-x 